\title{
Dynamic State Estimation of Electric Power System Integrating Wind Power Generation
}

\author{
Anna Glazunova ${ }^{1 *}$ \\ ${ }^{1}$ Melentiev Energy Systems Institute SB RAS, Lermontov str. 130, Irkutsk, Russia
}

\begin{abstract}
This paper is concerned with a problem of operation control of the electric power systems integrating wind farms. Dynamic state estimation is used to obtain reliable information about state variables and to promptly forecast the upcoming operating conditions. To predict the state variables, we assume that the wind farms are loaded first, and the remaining load is covered by traditional generation. Active power balance is maintained by a balancing plant. The problem of considering the information about wind speed at an upcoming time to forecast state variables is studied.
\end{abstract}

\section{Introduction}

Electric power systems (EPSs) have some level of flexibility. One of the flexibility characteristics is the EPS ability to constantly keep a balance between power consumption and production. The introduction of renewable energy sources in EPS makes it difficult to maintain power balance, due to the dependence of wind and solar generation on weather conditions. Such energy sources are uncontrollable. The number of uncontrolled energy sources that can be integrated into an EPS is determined by the degree of the EPS flexibility.

A significant part of renewable energy sources are wind farms. Placing wind farms into operation within power systems necessitates a study of their effect on:

- the Total Transfer Capability and Available Transfer Capability values [1], [2]; [3];

- $\quad$ optimal loading of EPS generating equipment [4];

- $\quad$ Electric Power System state estimation result [5], [6];

- $\quad$ and others.

In this paper, I apply dynamic state estimation (DSE) to obtain reliable information on state variables of EPS with wind farms. The research is focused on the dependence of the quality of estimates and forecasts of measurements, on different options of injection at wind farms.

The paper is organized as follows. The second section presents a problem statement. The third and fifth sections give information about measurements and about a technique for state estimation. The fourth and sixth sections describe modeling of active power generation at wind farm, wind speed and operating conditions. The seventh section is devoted to the application of this technique. The last section presents the conclusions.

This study is supported by the Siberian Branch of the Russian Academy of Sciences (Project III.17.4.2) of the Federal Program of Scientific Research (№ AAAA-A17-117030310438-1).

Corresponding author: glazunova@isem.irk.ru

\section{Problem statement}

Two assumptions are made in the research:

1. The wind farms are loaded first and the remaining load is covered by traditional generation. Active power balance is maintained by a balancing plant.

2. There is information about the wind speed in the area under consideration at the current and upcoming time.

The state variables are calculated in real time using the state estimation methods that employ on-line information (measurements) obtained from a measuring system. There are two options to take into account the wind farm output in the state estimation problem. They differ in availability of measuring devices at the wind farm.

In the first option, active and reactive power is represented by a model of measurements, and the wind farms are regarded as traditional energy sources.

The second case employs pseudo-measurements. Their values are calculated at the considered time instant and depend on the wind speed at this time instant and on the installed capacity.

- Maintaining the maximum power output conditions at wind farms requires rapid load redistribution between generators when there is no or insufficient wind to supply electricity to all consumers. This necessitates information on state variables some time in advance. According to the predicted data, control actions are taken to change power at balancing plant to maintain active and reactive power balance in the electric power system. The state variables are predicted using dynamic state estimation.

Table 1 shows the differences in dynamic state estimation for different options of injection at wind farm. 
Table 1. An enlarged scheme of the algorithm for dynamic state estimation

\begin{tabular}{|c|c|c|c|}
\hline & Option & 1 & 2 \\
\hline & & $\begin{array}{l}\text { Active and reactive power is } \\
\text { represented by a model of } \\
\text { measurements }\end{array}$ & $\begin{array}{c}\begin{array}{c}\text { Active and reactive power is represented by a model of pseudo- } \\
\text { measurements }\end{array}\end{array}$ \\
\hline \multicolumn{4}{|c|}{ Initial information } \\
\hline & $\begin{array}{l}\text { Vector of } \\
\text { neasurements }\end{array}$ & $\begin{array}{l}\bar{y}=\left(U_{i}, P_{i}\right. \\
\left.Q_{i}, P_{i j}, Q_{i j}, \delta_{i}\right)\end{array}$ & $\bar{y}=\left(\bar{y}, P_{\mathrm{W}}^{P M}, Q_{W}^{P M}\right)$ \\
\hline \multicolumn{2}{|c|}{$\begin{array}{l}\text { Model of wind } \\
\text { farm generation }\end{array}$} & $\bar{y}=y_{\text {true }}+\xi_{y}$ & $P_{W}=\left\{\begin{array}{cc}0, & x<V_{c i n} \\
P_{r}\left(A+B x+C x^{2}\right), & V_{c i n} \leq x<V_{r} \\
P_{r}, & V_{r} \leq x<V_{c o} \\
0, & x \geq V_{c o}\end{array}\right.$ \\
\hline & $\begin{array}{l}\text { Objective } \\
\text { function }\end{array}$ & $\begin{array}{l}J(x)=(\bar{y}-y(x))^{\mathrm{T}} R_{y}^{-1}(\bar{y}-y(x)) \\
+(\tilde{x}-x)^{T} M^{-1}(\tilde{x}-x)\end{array}$ & $\begin{array}{l}J(x)=(\bar{y}-y(x))^{\mathrm{T}} R_{y}^{-1}(\bar{y}-y(x))+ \\
(\tilde{x}-x)^{T} M^{-1}(\tilde{x}-x)+ \\
\left(P_{W}^{P M}-P_{W}(x)^{T} R_{W}^{-1}\left(P_{W}^{P M}-P_{W}(x)\right)\right.\end{array}$ \\
\hline \multicolumn{4}{|c|}{ Dynamic state estimation } \\
\hline & Action & \multicolumn{2}{|c|}{ Minimize objective function $\frac{\partial J}{\partial x}=0$ to calculate state vector } \\
\hline \multicolumn{2}{|r|}{$\begin{array}{l}\text { Nonlinear } \\
\text { system of } \\
\text { equations }\end{array}$} & $\begin{array}{c}H^{\mathrm{T}} R_{y}^{-1}(\bar{y}-y(x))=0, \\
M^{-1}(\tilde{x}-x)=0\end{array}$ & $\begin{array}{c}H^{\mathrm{T}} R_{y}^{-1}(\bar{y}-y(x))=0 \\
M^{-1}(\tilde{x}-x)=0 \\
H_{W}^{\mathrm{T}} R_{W}^{-1}\left(P_{W}^{P M}-P_{W}(x)\right)=0\end{array}$ \\
\hline & Action & \multicolumn{2}{|c|}{ Calculate state variable $z=z(x)$, where $z=\left(P_{i}, Q_{i}, P_{i j}, Q_{i j}\right)$} \\
\hline \multicolumn{2}{|r|}{ Result } & \multirow{2}{*}{\multicolumn{2}{|c|}{$\begin{array}{l}\text { Current state is estimated } \\
\text { Calculate state vector disregarding the information on wind speed at an upcoming time instant } \\
\qquad \tilde{x}_{k+1}=F_{k+1} \hat{x}_{k}+\Delta \tilde{x}_{k}\end{array}$}} \\
\hline & \multirow{2}{*}{$\begin{array}{l}\text { there is no } \\
\text { information } \\
\text { about wind } \\
\text { speed at the } \\
\text { upcoming } \\
\text { time instant }\end{array}$} & & \\
\hline & & \multicolumn{2}{|c|}{ Calculate state variables } \\
\hline & \multirow{2}{*}{$\begin{array}{l}\text { there is } \\
\text { information } \\
\text { about wind } \\
\text { speed at the } \\
\text { upcoming } \\
\text { time instant }\end{array}$} & \multicolumn{2}{|c|}{ 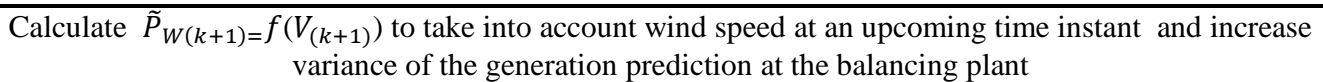 } \\
\hline & & \multicolumn{2}{|r|}{ State estimation } \\
\hline
\end{tabular}

In the sections to follow, the information from Table 1 is presented in detail.

\section{State estimation and vector of measurements}

The problems of analysis, control and scheduling of operation cannot use unprocessed measurements as input data because electric power system is insufficiently furnished with measurements and measurements contain errors. Processing of measurements, i.e. filtration of errors in measurements and calculation of unmeasured state variables, involves the methods of state estimation. According to how the data are processed, the state estimation methods can be divided into static and dynamic. 
The static state estimation implies calculation of a steady state by the data of measurements disregarding the interrelations between state variables changing in time. The dynamic state estimation is a calculation of steady state by the data of measurements given the interrelations between the state variables changing in time.

The main advantage of the dynamic state estimation is its capability to project state variables for a short time period, i.e. estimate the electric power system conditions in advance. Moreover, it decreases failures and losses of data in the measurement information. The first studies on state estimation started in the late 1960s [7], [8], [9].

State estimation of power system implies calculation of state variables, which is made on the basis of measurements obtained from Supervisory Control And Data Acquisition (SCADA) system and Wide-Area Measurement Systems (WAMS).

The measurements represent a sum of a true value $y_{\text {true }}$ and a normal noise $\xi_{y}$ :

$$
\bar{y}=y_{\text {true }}+\xi_{y}, \quad \xi_{y} \in N\left(0, \sigma_{y}^{2}\right),
$$

where $\sigma_{y}^{2}$ - measurement variance.

The vector of measurements looks as follows:

$$
\bar{y}=\left(U_{i}, \delta_{i}, P_{i_{i}}, Q_{i}, P_{i j}, Q_{i j_{i}}\right) \text {, }
$$

where $U_{i}$-magnitudes of voltages; $\delta_{i}-$ voltage phases, $P_{i}, Q_{i}$ - injections of active and reactive powers at nodes, $P_{i j}, Q_{i j}-$ power flows in transformers and lines.

\section{Modeling of active power generation at wind farm}

The relationship between the wind power generation and wind speed is nonlinear. The obtained power can be calculated from the curve "speed-power" [3], [10]. Figure 1 shows the curve that is used in this article.

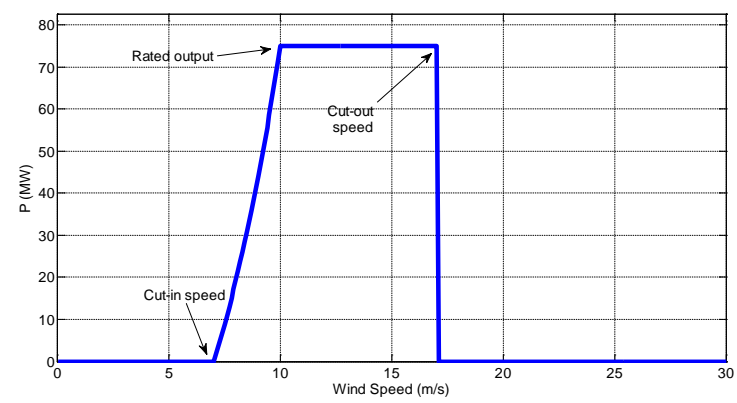

Fig.1. A curve of wind turbine

As can be seen from Figure 1, the wind turbine starts to work at wind speed "Cut-in speed" $\left(V_{c i n}\right)$ and reaches rated power at wind speed "Rated output" $\left(V_{r}\right)$. Then, despite the increase in wind, the turbine power does not increase until "Cut-out speed" $\left(V_{c o}\right)$ is reached.

This study is supported by the Siberian Branch of the Russian Academy of Sciences (Project III.17.4.2) of the Federal Program of Scientific Research (№ AAAA-A17-117030310438-1).

Corresponding author: glazunova@isem.irk.ru
The dependence of turbine power on wind speed in a mathematical form can be written as follows:

$$
P_{W}=\left\{\begin{array}{cc}
0, & x<V_{c i n} \\
P_{r}\left(A+B x+C x^{2}\right), & V_{c i n} \leq x<V_{r} \\
P_{r}, & V_{r} \leq x<V_{c o} \\
0, & x \geq V_{c o}
\end{array}\right.
$$

where A, B, C - are constants determined by the wind turbine parameters.

Equations (3) are used to calculate the amount of generated power. Reactive power of wind turbine depends on the type of turbine. Wind turbines can operate at a fixed or variable speed. The fixed -speed and limited -speed turbines use capacitor banks to compensate for reactive power, while variable-speed turbines can control voltage, active and reactive power. Fixed-speed turbine is considered as an ordinary PQ node where active and reactive power is constant. In this paper, active power is determined by the "speed-power" curve, and reactive power is calculated using a coefficient. With the pseudomeasurements of active $P_{W}^{P M}$ and reactive $Q_{W}^{P M}$ power injections at wind farm the vector of measurements looks as follows:

$$
\bar{y}=\left(U_{i}, P_{i}, Q_{i}, P_{i j}, Q_{i j}, \delta_{i}, P_{W}^{P M}, Q_{W}^{P M}\right) .
$$

\section{Dynamic state estimation}

The dynamic model applied determines largely the efficiency of dynamic state estimation. For a short-term forecasting of state parameters we use simple dynamic models. Based on a priori knowledge of the system behavior, the state space representation for a discrete time-variant system is described by the following equations

$$
\begin{gathered}
x_{k+1}=F_{k} x_{k}+\xi_{F(k)}, \\
\bar{y}_{k}=H_{k} x_{k}+\xi_{y(k)},
\end{gathered}
$$

where $\quad x_{k}$ - state vector at time instant $k, \quad F_{k}$ transition matrix from state $k$ to state $k+1, \bar{y}_{k}-$ measurements at time instant $k, \xi_{\mathrm{F}(k)}$ - model noise $\xi_{F(k)} \in N\left(0, \sigma_{F(k)}^{2}\right), \xi_{y_{k}}-$ measurement error, $k-$ snapshot number. State vector $x=(\delta, U)$ has a dimension $2 \mathrm{n}$, where $\mathrm{n}$ is the number of nodes in the calculated scheme. Such a state vector determines all the other state parameters.

In this research, the extended Kalman filter is used for the dynamic state estimation [11], [12].

After some transformations of equations (5), (6) we can obtain a covariance matrix of forecast errors:

$$
M_{k+1}=F_{k} P_{k} F_{k}^{T}+W_{F(k)},
$$

where $W_{F(k)}$ - the covariance matrix of dynamics model noise consists of $\sigma_{F(k)}^{2}, P_{k}$ - covariance matrix of the state vector component estimates. Elements of matrix 
$F_{k}$ are determined in advance by retrospective information.

The objective function in the dynamic state estimation looks as follows:

$$
J(x)=(\bar{y}-y(x))^{\mathrm{T}} R_{y}^{-1}(\bar{y}-y(x))+(\tilde{x}-x)^{T} M^{-1}(\tilde{x}-x),
$$

where $\tilde{X}$ - forecast of state vector components.

In order to calculate the vector of state, the derivative $\frac{\partial J(x)}{\partial x}$ is equated to zero, and the system of non-linear equations is solved:

$$
\frac{\partial J(x)}{\partial x}=H^{T} R^{-1}(\bar{y}-y(x))+M^{-1}(\tilde{x}-x) .
$$

Due to nonlinear dependence $y(x)$, the problem is solved iteratively by Newton's method. The system is linearized at each iteration and a system of linear equations is solved, where correction vector is calculated by the equation:

$$
\begin{gathered}
\Delta x^{(i)}=P^{i}\left(H^{T(i)} R_{y}^{-1}\left(\bar{y}-y\left(x^{i}\right)\right)+M^{-1}\left(\tilde{x}-x^{i}\right)\right), \\
P^{i}=\left(H^{T(i)} R^{-1} H^{i}\right)^{-1},
\end{gathered}
$$

where $i$ - iteration number.

Components of the state vector are calculated by the equation:

$$
x^{i+1}=x^{i}+\Delta x^{i} .
$$

If $\Delta x^{i}<d_{x}$, the iteration process has converged, where $d_{x}$ - accuracy of the iterative process, and all state variables are calculated.

The following equation is used in order to forecast the state vector components:

$$
\begin{aligned}
& \tilde{x}_{k+1}=F \hat{x}_{k}+\Delta \tilde{x}_{k}, \\
& \Delta \tilde{x}_{k}=P_{k} M_{k+1}^{-1}\left(\tilde{x}_{k}-\bar{x}_{k}\right) .
\end{aligned}
$$

The forecast error $\Delta \tilde{x}_{k}$ in the first forecasting cycle is determined by the equation:

$$
\Delta \tilde{x}_{k}=P_{k} M_{k+1}^{-1}\left(\hat{x}_{k-1}-\bar{x}_{k}\right),
$$

where $\hat{x}_{k}$ - estimates of state vector components.

The state vector is used to calculate all forecast state variables.

In the line 'forecast' in Table 1, we write the actions to be performed to forecast state variables. First, the forecast values of the state variables are calculated disregarding information about the wind speed at the upcoming time instant. Then the pseudo-measurement of active power generation at the wind farm is calculated taking into account the information about the wind speed at the upcoming time, the variance of the active power forecast value at the balancing plant is increased, and the dynamic state estimation is performed.

\section{Modeling of wind speed and operating conditions}

\subsection{Modeling of wind speed}

Fig.2 shows a wind speed profile.

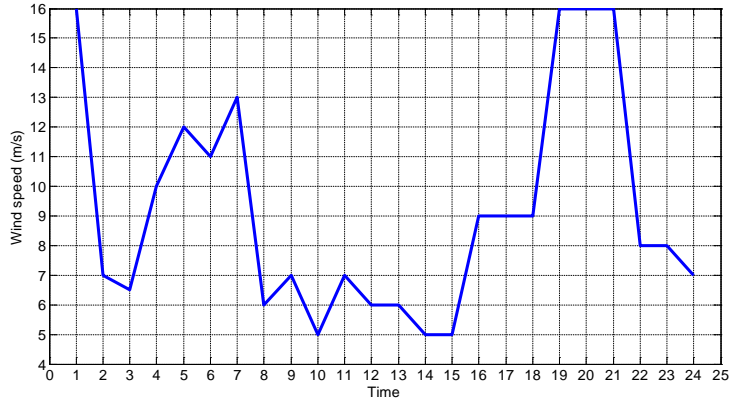

Fig.2. Wind speed profile

The simulations are carried out for wind speeds going from 7 to $17 \mathrm{~m} / \mathrm{s}$. The wind speed is assumed to be constant for $60 \mathrm{~s}$.

\subsection{Modeling of operating conditions}

An archive of conditions is created on the basis of the mathematical model of IEEE 30 test network under various operating conditions. Such a variety can be achieved by applying models of typical load profiles at chosen nodes. In this research, the load of residential consumer (city with a population of 20-250 thousand people) and load profile for two-shift industrial facility are modeled (Fig. 3).

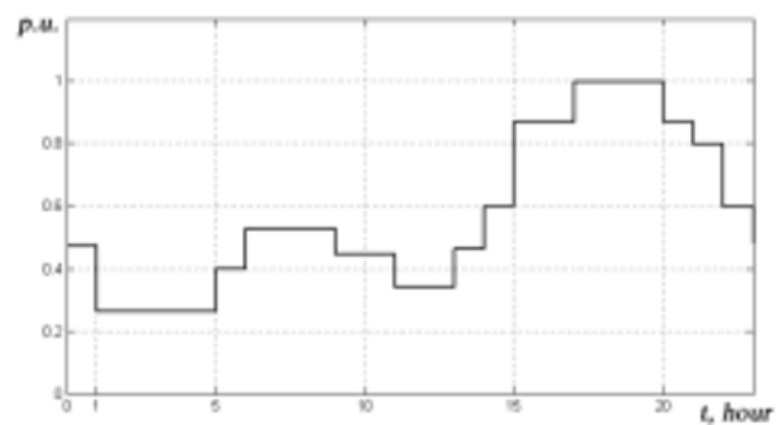

Fig. 3a. Load profile for residential consumer (city with population of 20-250 thousand people)

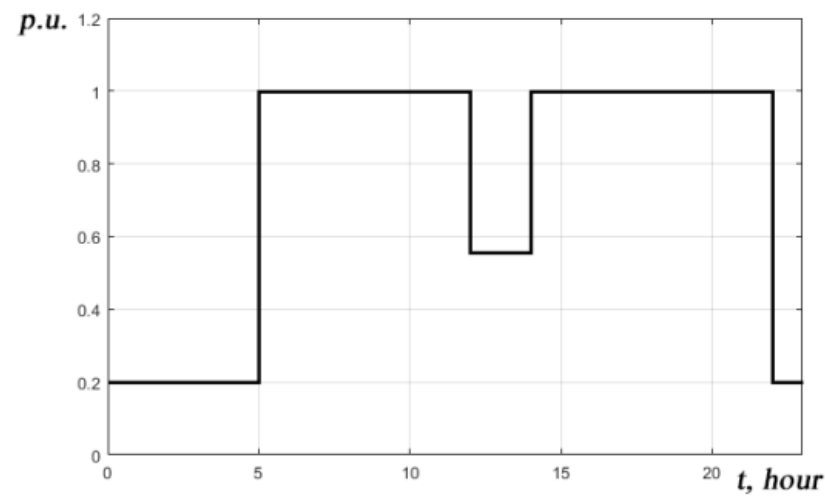

Fig. 3b. Load profile for two-shift industrial facility

Load flow solutions that are obtained 24 times correspond to each point in load curves. These solutions are then used to create measurement snapshots. Chosen state variables of a particular solution are all shifted by a 
small random value according to the model of measurement

$$
\bar{y}=y_{\text {true }}+x_{\text {rand }} \sigma_{y} \text {, }
$$

where $\sigma_{y}$ - measurement variance, $x_{\text {rand }}$ - random errors, generated by random number generator $x_{\text {rand }} \in N(0,1), \quad y_{\text {true }}$ - reference .

Each solution can be used several times in a row to form the longevity of an unchanging state. In this research each solution was used 10 times to form 240 snapshots of measurements.

\section{Case study}

The calculations are performed on the IEEE 30 Bus Test Case (Fig. 4) according to the scenario: calculate steady-state parameters with the help of dynamic state estimation at the current and upcoming time instant.

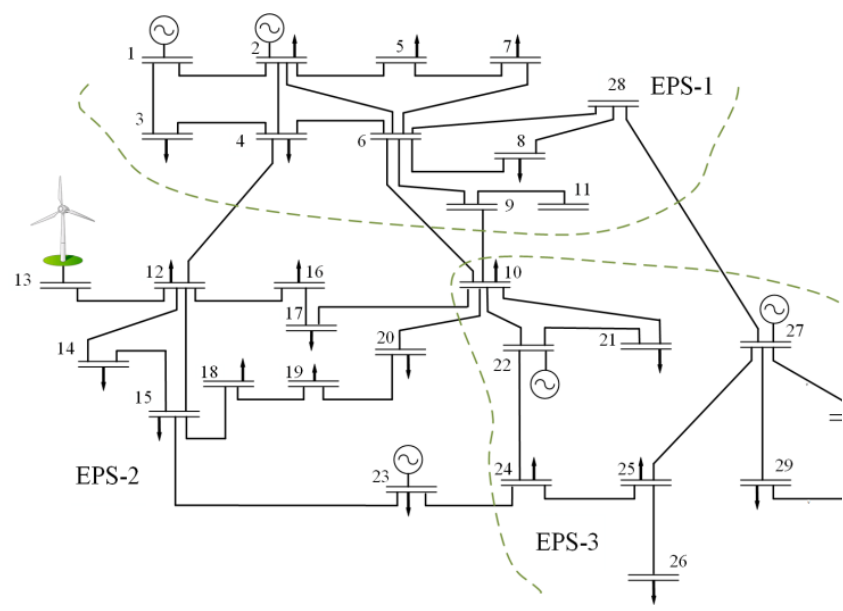

Fig 4. The IEEE 30 Bus Test Case

When forecasting the parameters, take into account either presence or absence of information about the wind speed at the upcoming time instant. The wind farm is denoted by node 13 . The balancing node is node number 2. At node 21 typical load profiles (fig.3) is modeled. Calculations are made for option 1.

Fig. $5 a$ and $5 b$ show the values of active power generation at the wind and balancing stations in 24 and in 240 various operating conditions.

Calculation 1. The initial data for the calculation of steady state at the current time instant are: measurements and variances of measurements.

The initial data for calculation of steady state at the upcoming time point are: forecasts of the state vector components, and the covariance matrix of the forecast errors.

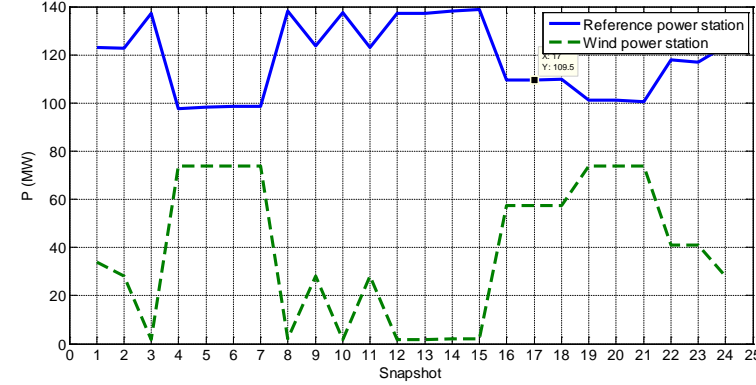

a). 24 operating conditions

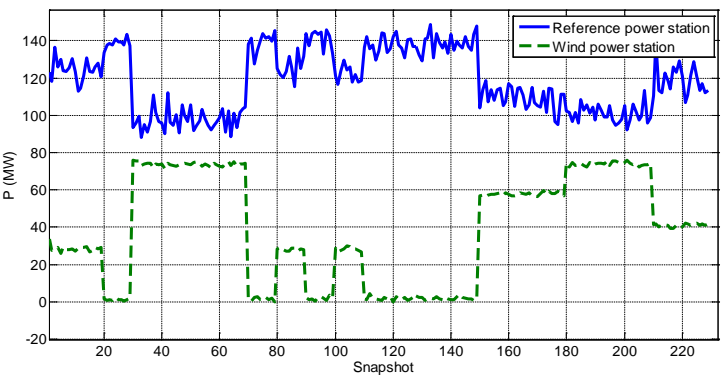

б). 240 operating conditions

Fig.5. Active power injection at wind farm and at slack node

Calculation 2. The initial data for the calculation of steady state at the upcoming time point are: forecast state variables, covariance matrix of forecast errors, active and reactive power injections at wind farms (calculated from (3)), variances of pseudo-measurements, and variances of active power injection forecasts at the balancing plant.

Fig. 6 demonstrates the measurements, estimates and forecasts of active power injection at node 13, in case we don't take into account the wind speed information at an upcoming time.

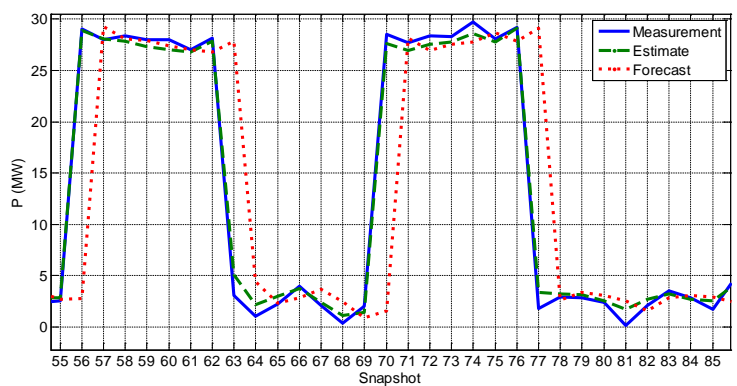

Fig.6. Measurements, estimates and forecasts of active power injection at node 13

Fig. 6 shows that a forecast is incorrectly calculated in case a profile of curve is changed.

Figure 7 shows measurements, estimates and forecasts of active power generation at node 13, taking into account the wind speed information at an upcoming time to calculate active power generation at the wind farm. 


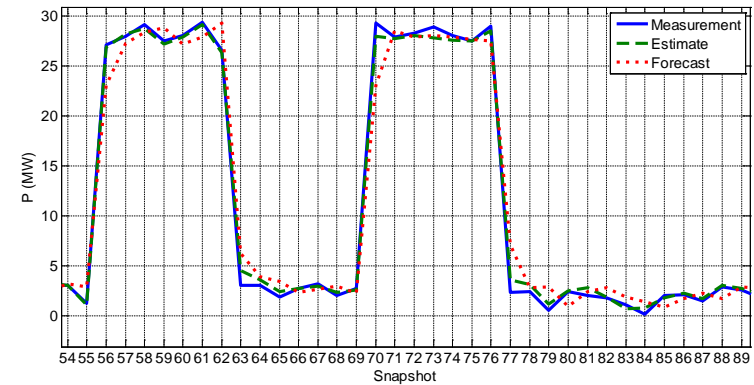

Fig.7. Measurements, estimates and forecasts of active power injection at node 13 , taking into account the wind speed information at an upcoming time

Figure 7 shows that a forecast is correctly calculated in case a profile of curve is changed.

\section{Conclusion}

The paper describes dynamic state estimation of an electric power system with wind farms. The research is focused on two options of considering the information on the wind farm output in dynamic state estimation in electric power system. In the first option, the information about generation is represented by measurements, in the second option - by pseudo-measurements. Values of pseudo-measurements are calculated depending on the wind speed and the installed capacity of the wind farm.

Modeling of EPS operating conditions and measurement snapshots and wind speed profile was performed. The operating conditions were modeled using load curves at load nodes.

The analysis of the dynamic state estimation results for a power system with wind farms demonstrates that the forecast of state variables is more accurate when based on the forecast of pseudo-measurements. The forecast data are used to generate control actions at the balancing plant to maintain the active and reactive power balance in the electric power system.

\section{References}

1. Xiaochen Zhang, James Jamal Thomas, Santiago Grijalva, Transmission and Distribution Conference and Exposition ( 2016)

2. Yunfei Chu, Zhinong Wei, Guoqiang Sun, Yichi Li, 5th International Conference on Electric Utility Deregulation and Restructuring and Power Technologies (DRTP) (Changsha, China, 2015)

3. Hamid Falaghi, Maryam Ramezani, Chanan Singh, Mahmood-Reza Haghifam, IEEE Systems Journal, 6, 1, 181-190 (2012)

4. B. C.Ummels, M.Gibescu Pelgrum, W. L Kling., A. J.Brand, IEEE Trans. Energy Conversion, 22, 1, 44-51 (2007)

5. Yu Shenglong, Kianoush Emami, Tyrone Fernando, H. C. Iu Herbert, Kit Po Wong, IEEE Transactions on Power Systems, 31, 6, 4935-4944 (2016)
6. SUN Guo-qiang, WEI Zhi-nong, PANG Bo (2009)

7. F.S.,Schweppe J. Wildes, Power Apparatus and Systems, 89, 1, 120-125 (1970)

8. F.S.Schweppe, D.B.Rom Power Apparatus and Systems, 89, 1, 125-130 (1970)

9. A.Z.Gamm, in Issues of estimation and identificaiton in energy systems (Irkutsk, SEI SB AS USSR, 1974).

10. Nishad Mendis, Kashem Muttaqi, Saad Sayeef, Sarath Perera, in IEEE Power and Energy Society General Meeting (Wollongong, 2010)

11. M.B. Do Coutto Filho, J.C. Stacchini de Souza, R.S. Freund Power System, 24, 4, 1667-1677 (2009)

12. Hadis Karimipour, Venkata Dinavahi, Smart grid, 6, 3, 1539-1549 (2015) 